\title{
Determining the Influence of Pore Shape on Filtering Quality of Spunbonded Nonwovens
}

\author{
G. X. JIN ${ }^{1,2, a^{*}}$, Q. Z. LI $\left.\right|^{1, b}$, C. Y. ZHU ${ }^{1, c}$ \\ ${ }^{1}$ College of Materials and Textiles, Zhejiang Sci-Tech University, Hangzhou 310018, China \\ ${ }^{2}$ Zhejiang Industry Polytechnic College, Shaoxing 312000, China \\ actljgx@163.com, bliqizheng@139.com, chengyanzhu@126.com \\ *Corresponding author: ctljgx@163.com
}

Keywords Spunbonded; nonwovens; pore shape; filtering quality; digital image processing

\begin{abstract}
The pore shape of spunbonded nonwovens and its influence on the filtering quality were investigated in this study. An index named the filtration index (FI) was first proposed to characterize the performance of the higher filtration efficiency against the lower pressure drop of spunbonded nonwovens. A parameter called plump degree was used to characterize the pore shape and was measured by applying digital image processing technology. The effect of pore plump degree on FI was discussed. The results show that FI decreases with the increase of pore plump degree and showed a significant linear dependence (correlation coefficient $\mathrm{R}=0.96684$ ). This work can provide a theoretical foundation for the development of multilayer composite nonwovens air filter materials with high filtering quality.
\end{abstract}

\section{Introduction}

There has been an increasing demand for the filtration of particulate matter smaller than $2.5 \mu \mathrm{m}$ (PM2.5) due to atmospheric pollution. Air filtration materials should demonstrate high filtering quality, which means higher filtration efficiency against lower pressure drop. Nonwoven fabric is an aggregation consisting of two parts: the fiber and the pore. As an ideal filter material, nonwovens have been widely applied in air filtration. The performance of a material is determined by its structure. The filtration performance of nonwoven is closely related with its fiber web structure including the pore structure [1-3]. The pore structure consists of the porosity, pore shape, pore size, and pore size distribution. Abundant research on pore structure has been conducted, and most of these studies have focused on the porosity, pore size, and pore size distribution [4-8]. Our literature review reveals that only a few studies have evaluated the pore shape of nonwovens [9-12]. However, the pore shape has not been considered to be an important factor that would affect the filtering quality of the materials. Though spunbonded nonwovens rarely serve as the sole component of an air filter, it is an ideal composite base material due to its excellent mechanical performance, short processing flow, and low manufacturing cost. In this study, the filtration index (FI) was proposed to characterize the filtering quality of air filter materials. A parameter called plump degree was used to characterize the pore shape in spunbonded nonwovens, and its influence on filtering quality was investigated. Exploring the influence of pore shape on the filtering quality of thin monolayer nonwovens can provide a theoretical foundation for the development of multilayer composite nonwoven air filter materials with high filtering quality.

\section{Experimental Procedure}

\section{Definition of the Filtration Index}

Filtering quality is usually expressed by the "quality factor" $\left(q_{F}\right)$, which is defined as ${ }^{[13]}$

$$
q_{F}=\frac{\ln (1 / P)}{\Delta p}
$$

Where $P$ is the fraction of aerosol penetrated and $\Delta p$ is the pressure drop. However, $P$ is a dimensionless index (percentage), whereas $\Delta p$ is conventionally given in millimeters of $\mathrm{H}_{2} \mathrm{O}$. Thus, 
$\Delta p$ should be non-dimensionalized prior to the calculation of filtering quality. In this study, the efficacy coefficient method was used to evaluate the filtering quality of material ${ }^{[14]}$. The detailed calculation is given as follows.

Assume that there is a matrix $\mathrm{A}=\left(a_{i j}\right)$, where $a_{i j}$ is the actual value of filtration performance. Here, $i$ is the sample number, while $j$ indicates the type of filtration performance. If $j=1, a_{i j}$ is the value of filtration efficiency. If $j=2, a_{i j}$ refers to the value of the pressure drop. First, matrix A should be non-dimensionalized. The resulting dimensionless matrix is expressed by $R=\left(r_{i j}\right)$, where

$$
r_{i j}=a_{i j} / \sqrt{\left(\sum_{i=1}^{n} a_{i j}{ }^{2}\right)}
$$

The method to calculate the efficacy coefficient of $a_{i j}$ depends on the filtration performance. Higher filtration efficiency values indicate better performance, so the efficacy coefficient of the filtration efficiency must be calculated according to

$$
d_{i 1}=\frac{r_{i 1}}{r_{\max 1}}
$$

where $r_{\max 1}$ is the maximum of the non-dimensionalized value of the filtration efficiency. However, lower pressure drop values indicate better performance; therefore, the efficacy coefficient of pressure drop should be calculated using

$$
d_{i 2}=1+\frac{r_{\min 2}-r_{i 2}}{r_{\max 2}}
$$

where $r_{\max 2}$ is the maximum of the non-dimensionalized value of the pressure drop and $r_{\min 2}$ is the minimum of the non-dimensionalized value of the pressure drop. Hence, the efficacy coefficient matrix can be obtained, expressed as $D=\left(d_{i j}\right)$. The filtration index $(F I)$ was first proposed to characterize the filtering quality of spunbonded nonwovens, where FI can be calculated using

$$
F I_{i}=u_{1} d_{i 1} \times+u_{2} \times d_{i 2}
$$

where $i$ is the sample number, and $u_{1}$ and $u_{2}$ are the weight coefficients of the filtration efficiency and pressure drop, respectively. In this study, $u_{1}=u_{2}=0.5$.

\section{Acquisition of Spunbonded Nonwoven Samples}

Five samples of polypropylene spunbonded nonwoven, which were acquired from the Key Laboratory of Advanced Textile Material and Manufacturing Technology, Ministry of Education, Zhejiang Sci-Tech University, were selected. The basis weight and thickness of the samples are shown in Table 1.

Table 1 The basis weight and thickness of the spunbonded nonwovens samples

\begin{tabular}{|c|c|c|}
\hline Sample No. & Basis weight $\left(\mathrm{g} \cdot \mathrm{m}^{-2}\right)$ & Thickness $(\mathrm{mm})$ \\
\hline 1 & 127.7 & 0.604 \\
\hline 2 & 109.8 & 0.579 \\
\hline 3 & 96.3 & 0.528 \\
\hline 4 & 82.8 & 0.490 \\
\hline 5 & 74.4 & 0.473 \\
\hline
\end{tabular}

\section{Test Method of Filtration Performance}

A SX-L1050 filtration efficiency tester (Suxin Purification Equipment Company, Ltd., Suzhou, China) was used to measure the filtration efficiency and the pressure drop on spunbonded nonwoven samples in this study. The test flow was $32 \mathrm{~L} / \mathrm{min}$; the filtering rate was $5.33 \mathrm{~cm} / \mathrm{s}$; the upstream dilution multiple ranged from 7 to 10 times; the size of the monodispersed polystyrene (PS) emulsion was $\geq 0.3 \mu \mathrm{m}$; and the area of the sample was $100 \mathrm{~cm}^{2}$. On each sample, five places were tested, and the averages were determined. 


\section{Characterization and Determination of Pore Shape}

The movement of aerosol particles in filtering media may occur as a result of diffusional deposition (the diffusion distance per second of a $0.1-\mu \mathrm{m}$ particle can be up to $17 \mu \mathrm{m}$ at room temperature), inertial impaction, interception, gravity, or electric forces ${ }^{[15-17]}$. All of the above reasons describe how materials may filter particles that are significantly smaller than the size of pores. However, from another point of view, we can also assume that a particle will pass through the filter only if it has a diameter of a certain minimum circumscribed circle (including equivalent circles resulting from the diffusion effect) that is smaller than the diameter of the maximum inscribed circle of the pore. In what follows, we consider whether the pore shape has any effect on the filtering quality of air filter material using an ellipse-shaped pore as an example. Assume that there are two ellipse-shaped pores, pore A and pore B, as shown in Fig.1. The two pores have the same area. However, the eccentricity of pore $B$ is larger than that of pore $A$. As a result, the maximum inscribed circle of pore A is obviously larger than that of pore B. Therefore, pore B can intercept more particles than pore A, even though the two pores have the same air permeability. Therefore, we can deduce that the FI value of pore B is higher than that of pore A.

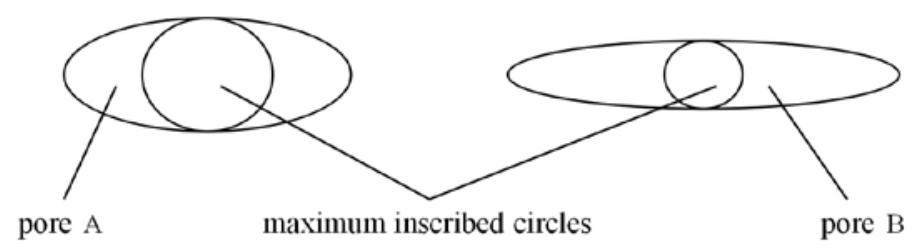

Figure 1 Ellipse-shaped pores with different eccentricities

According to the above hypothesis, a relationship could exist between the FI value of the filter material and its pore shape, particularly in the case that the material is a thin nonwoven fabric. However, the actual pore shape of nonwoven fabric is much more complex than the ellipse-shaped pores considered above. In this study, we use a parameter called plump degree $\left(D_{P}\right)$ to characterize the pore shape. It is defined as the ratio of the area of the pore's maximum inscribed circle to the area of the pore itself:

$$
D_{p}=\frac{A_{\text {maxic }}}{A_{\text {pore }}}
$$

where $A_{\text {maxic }}$ is the area of the pore's maximum inscribed circle and $A_{\text {pore }}$ is the pore area. For the same pore area, a larger plump degree value indicates that the inner space of the pore is more spacious. A JSM-5610LV scanning electron microscope (JEOL Company, Ltd., Tokyo, Japan) was used to collect the scanning electron microscopy (SEM) images of the samples with an amplifying multiple of 200. Ten different places were scanned on each sample; thus, a total of 50 images were collected. Fig. 2(a) shows the SEM image No. 1 of sample No. 1 for an image size of $400 \times 400$ pixels. 


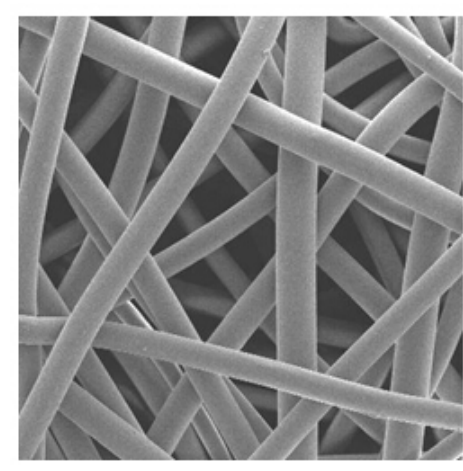

(a)

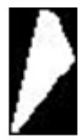

(c)

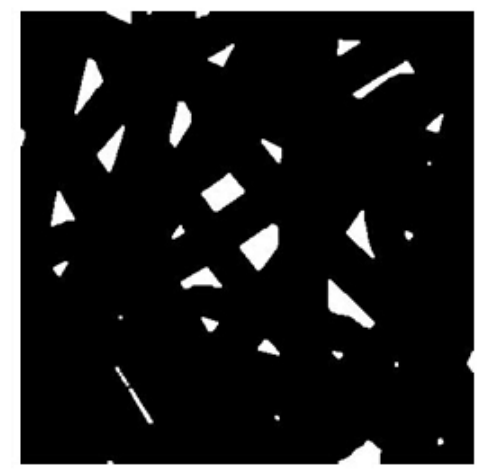

(b)

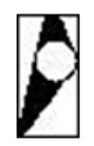

(d)

Figure 2 Determination of the maximum inscribed circle of a pore (a) SEM image No. 1 of sample No. 1, (b) Preprocessed image No. 1 of sample No. 1, (c) The extracted image of pore No. 4, (d) The maximum inscribed circle of pore No. 4

In order to obtain an image with a distinct pore shape and boundary, a number of digital image processing techniques, including histogram processing, median filtering, binarization, and mathematical morphology processing, was employed using Matlab programming. Fig. 2(b) shows the preprocessed image No. 1 of sample No. 1. A cell array, which could also be called a generalized matrix, was established based on the pore labeling, so as to calculate the plump degree value of each pore. Here, each cell is a sub-matrix corresponding to a pore. After the incomplete pores located on the edge of the image were excluded, 1652 pores were extracted from the SEM images of the five considered samples. For example, Fig. 2(c) shows the pore No. 4. The radius of the pore's maximum inscribed circle was calculated using the "extreme value distance method," which is described as follows. First, the distances between an arbitrary point inside the pore and each point of the boundary were calculated using MATLAB functions, and the minimum value among these distances is then selected. For each point inside the pore, the corresponding minimum distance can be obtained. Thus, the maximum value among them is the radius of the pore's maximum inscribed circle. Fig. 2(d) shows the maximum inscribed circle of pore No. 4.

\section{Results and Discussion}

Table 2 shows the test results of filtration efficiency and pressure drop on the five samples.

Table 2 Filtration performance of the samples

\begin{tabular}{|c|c|c|}
\hline $\begin{array}{c}\text { Sample } \\
\text { No. }\end{array}$ & $\begin{array}{c}\text { Filtration } \\
\text { efficiency } \\
(\%)\end{array}$ & $\begin{array}{c}\text { Pressure } \\
\text { drop } \\
(\mathrm{Pa})\end{array}$ \\
\hline 1 & 31.4578 & 11.04 \\
\hline 2 & 28.4121 & 9.14 \\
\hline 3 & 26.3823 & 7.71 \\
\hline 4 & 24.3162 & 5.88 \\
\hline 5 & 21.3693 & 3.72 \\
\hline
\end{tabular}


The FI values of the five samples were calculated according to Table 2 using the efficacy coefficient method. In addition, the mean value of pore plump degree for each sample can be calculated according to the measurement results based on the method, which was introduced in Chapter 2.4. Using Origin software, the relationship between the pore plump degree and FI was analyzed by fitting the corresponding data, i.e., the values of FI and the average values of the pore plump degree for all five samples. The result is shown in Fig.3. We find a good correlation between FI and the pore plump degree. With an increase of the pore plump degree, FI will decrease. This relationship is given by the linear fitting equation

$$
y=1.30447-1.48878 x
$$

The correlation coefficient $\mathrm{R}=0.96684$, which means the linear curve fit is good. This result confirms that the pore shape will affect the filtering quality of nonwovens. Therefore, the pore shape should be taken as an important factor in the development of multilayer composite nonwoven air filter materials, so as to improve their filtering quality.

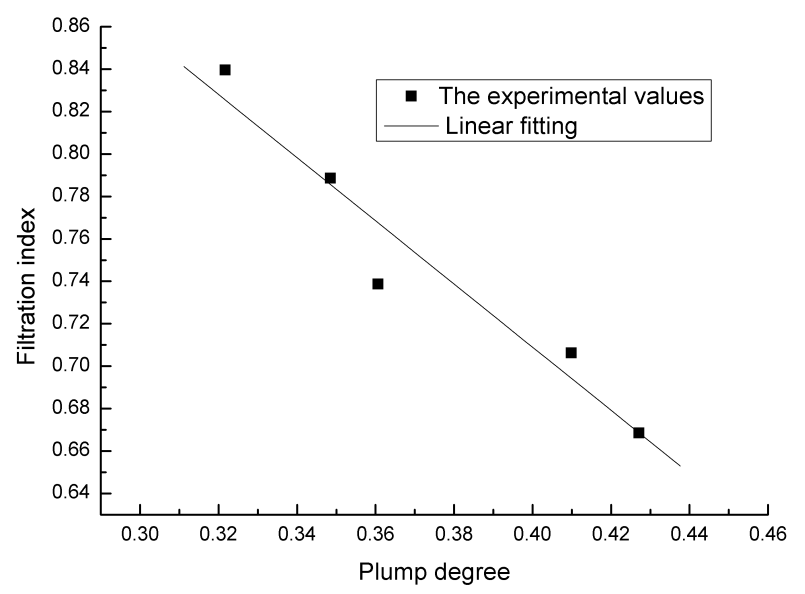

Figure 3 Relationship between the pore plump degree and FI

\section{Conclusion}

The filtration efficiency or the pressure drop of nonwovens is determined by structural parameters such as thickness, fiber diameter, and fiber shape. However, the pore shape could influence the filtering quality of the material. The results of the experiment show that, with the increase of the pore plump degree, the filtration index of the spunbonded nonwoven will decrease. In this study, the influence of pore shape in spunbonded nonwovens on its filtering quality was investigated. However, the formation mechanism of the pore shape, particularly the influence of processing parameter on the pore shape, was not discussed and will be considered in future research.

\section{Acknowledgment}

This work was supported by a grant from the Key Laboratory of Industrial Textile Material Preparation Technology Research of Zhejiang Province [2012E10010].

\section{References}

[1]Mazraeh-shahi, Z.T., Shoushtari, A.M., Bahramian, A.R. \& Abdouss, M., Synthesis, structure and thermal protective behavior of silica aerogel/PET nonwoven fiber composite. Fibers and Polymers, 15(10), pp. 2154-2159, 2014.

[2] Das, D., Das, S. \& Ishtiaque, S.M., Optimal design of nonwoven air filter media: effect of fiber Shape, Fibers and Polymers, 15(7), pp. 1456-1461, 2014.

[3] Jankowski, T., Influence of structural characteristics on liquid aerosol filtration in multilayer 
Nonwoven fabrics of the spunlace type, Fibres \& Textiles in Eastern Europe, 17(4), pp. 87-92, 2009.

[4] Aydilek, A.H., Oguz, S.H. \& Edil, T.B., Constriction size of geotextile filters, Journal of Geotechnical and Geoenvironmental Engineering, 131(1), pp. 28-37, 2005.

[5] Aydilek, A.H., Oguz, S.H. \& Edil, T.B., Digital image analysis to determine pore opening size Distribution of nonwoven geotextiles, Journal of Computing in Civil Engineering, 16(4), pp. 280-290, 2002.

[6] Simmonds, G.E., Bomberger, J.D. \& Bryner, M.A., Designing nonwovens to meet pore size Specifications, Journal of Engineered Fibers and Fabrics, 2(1), pp. 1-15, 2007.

[7] Gao, X.Y., Zhang, L. and Pan, Z.J., Porous characteristics and filtration properties of electrospun PA6 fiber composite, Journal of Textile Research, 31(1), pp. 5-10, 2010.

[8] Kothari, V.K., Agarwal, G.., Determination of pore size parameters and its distribution ofhydroentangled fabrics by image processing, Journal of Textile Institute, 99(4), pp.317-324, 2008.

[9] Lawrence, C.A., Shen, X., An investigation into the hydraulic properties for application in wet-press concrete casting, Part II: Predictive models for the water permeability of Needle-punched nonwoven fabrics, Journal of Textile Institute, 91(1), pp. 61-77, 2000.

[10] Amit, R., Structural analysis of pore size distribution of nonwovens, Journal of Textile Institute, 101(4), pp. 350-359, 2010.

[11] Yang, S., Yu, W.D., Pan, N., Study on texture character and acoustic absorbent behavior of Nonwovens, Technical Textiles, 7, pp. 6-11, 2010.

[12] Yang, X.H., Li, D.G., Quantitative expression of nonwovens’ pore structure, Technical Textiles,1, pp. 10-15, 2005.

[13] Han, D.H., Performance of respirator filters using quality factor in korea, Industrial Health, 38, pp. 380-384, 2000.

[14] Peng, F., Yuan, W., Hui, Z.Q., Research on exponential effective function for comprehensive evaluation, Statistical Research, 24(12), pp. 29-34, 2007.

[15] Julien, P., Philippe,V., Maryline, L., Anne, P., Sandrine, C.C. \& Dominique T., Influence of fiber diameter, fiber combinations and solid volume fraction on air filtration properties in nonwovens, Textile Research Journal, 82(19), pp. 1948-1959, 2012.

[16] Fu, H.M. Shen, H.G.., Research and development of the theory on air filtration, Journal of Filtration \& Separation, 13(3), pp. 20-24, 2003.

[17] Brown, R.C., Theory of airflow through filters modeled as arrays of parallel fibers, Chemical Engineering Science, 48(20), pp. 3535-3543, 1993. 